\title{
Computed tomography-guided core needle biopsy for lung nodules: low-dose versus standard-dose protocols
}

\author{
Yi-Yang Huang ${ }^{1}$, Hong Cheng ${ }^{1}$, Guang-Chao Li $^{2}$ \\ ${ }^{1}$ Medical College, Yangzhou University, Yangzhou, China \\ ${ }^{2}$ Department of Radiology, No. 6 Hospital of Shanghai Jiaotong University, Shanghai, China
}

Videosurgery Miniinv 2021; 16 (2): 355-361 DOI: https://doi.org/10.5114/wiitm.2021.103303

\begin{abstract}
Introduction: Computed tomography (CT)-guided core needle biopsy (CNB) is an essential step in the management of lung nodules (LNS). Low-dose CT (LDCT)-guided CNB has been used to decrease the radiation exposure.

Aim: To evaluate the technical success, safety, diagnostic capacity, and radiation exposure to patients between LDCT-guided and standard-dose CT (SDCT)-guided CNB for LNs.

Material and methods: This is a retrospective, single-centre study. Patients who underwent LDCT-guided or SDCT-guided CNB for LNs from January 2015 to December 2017 were included. Data on technical success, diagnostic performance, complications, and radiation exposure were collected and analysed.

Results: A total of 70 and 65 patients underwent LDCT-guided and SDCT-guided CNB procedure, respectively. The technical success rates were 100\% in both groups. The diagnostic yield, sensitivity, specificity, and overall diagnostic accuracy in the LDCT and SDCT groups were $71.4 \%$ and $67.7 \%(p=0.637), 97.8 \%$ and $93.2 \%(p=0.625), 100 \%$, and $100 \%$, and $98.6 \%$ and $95.4 \%(p=0.560)$, respectively. The independent risk factor of diagnostic failure was less sample tissues ( $p=0.012 ; 95 \%$ confidence interval: 0.033-0.651). Pneumothorax was found in 9 and 12 patients in the LDCT and SDCT groups, respectively $(p=0.369)$. Lung haemorrhage was found in 11 and 12 patients in the LDCT and SDCT groups, respectively $(p=0.671)$. The mean dose-length product was $38.3 \pm 17.0 \mathrm{mGy} \cdot \mathrm{cm}$ and 376.0 $\pm 118.7 \mathrm{mGy} \cdot \mathrm{cm}$ in the LDCT and SDCT groups, respectively $(p<0.001)$.

Conclusions: Compared to SDCT, LDCT-guided CNB can provide comparable safety and diagnostic performance for $L N s$ while reducing exposure to radiation.
\end{abstract}

Key words: low-dose, computed tomography, lung nodule, biopsy.

\section{Introduction}

Computed tomography (CT)-guided core needle biopsy (CNB) is widely used in the diagnosis of lung nodules (LNs). It has a diagnostic yield rate of $62-75 \%$ and an overall rate of diagnostic accuracy of $93-97 \%$ [1-3]. A LN is defined as a non-transparent $\leq 30 \mathrm{~mm}$ lesion that is surrounded by lung parenchyma [1-3]. In comparison to lung masses, LNs are smaller in size. Therefore, CT-guided CNB for LNs usually require ex- haustive scanning to adapt to the needle tip position. Hence, patients may be exposed to more radiation.

In recent years, low-dose CT (LDCT)-guided lung biopsy has gained popularity due to its reduced intra-operative radiation exposure [4-8]. However, most of the studies on LDCT-guided CNB included both lung masses and LNs. The inclusion of both disease types, despite their great size differences and therefore vast differences in radiation exposure, may have introduced potential bias. As such, it was cru-

Address for correspondence

Guang-Chao Li, Department of Radiology, No. 6 Hospital of Shanghai Jiaotong University, Shanghai, China, e-mail: ligch06@126.com 
cial to conduct a LDCT study using one unique disease type. Thus far, little is known about the efficacy of using LDCT-guided CNB for the diagnosis of LNs.

\section{Aim}

This study aimed to compare the technical success, safety, diagnostic performance, and radiation exposure of LDCT-guided and standard-dose CT (SDCT)-guided CNB for the diagnosis of LNs.

\section{Material and methods}

This retrospective research was approved by the Institutional Review Board of our hospital. The requirement for written consent from the participants was waived due to the retrospective nature of the study design.

\section{Patients}

From January 2015 to December 2017, consecutive patients underwent CT-guided CNB for LNs in our hospital. From January 2015 to October 2016, the SDCT-guided CNB was performed. From November 2016 to December 2017, LDCT-guided CNB was used. The decision to carry out a biopsy in these patients was dependent on the tenets of multidisciplinary diagnostic evaluation and aberrant results obtained upon chest CT examination [9].

The study inclusion criteria were as follows: (a) definite LN on CT; (b) LN size $\leq 30 \mathrm{~mm}$; (c) solid LN (> 80\% solid component of the total nodule); and (d) no definitive pathological diagnosis. The exclusion criteria were: (a) a shrunken $L N$ or a stable lesion (in size) over 2 years; (b) $L N$ size $\leq 5 \mathrm{~mm}$; and (c) patients with severe dysfunction of the lungs, heart, or kidneys or coagulation.

\section{Scanning parameters}

The CT instrument was a 16-row CT (Philips, Cleveland, Ohio). The same scanning parameters of both LDCT and SDCT included the following: tube voltage $=120 \mathrm{kV}$, thickness $=2 \mathrm{~mm}$, and collimation $=16 \times 0.75 \mathrm{~mm}$. The tube current in LDCT and SDCT was 15 and $150 \mathrm{~mA} / \mathrm{s}$, respectively.

\section{Computed tomography-guided core needle biopsy procedure}

All CT-guided CNB procedures were conducted by an experienced chest radiologist with more than
5 years of experience of CT-guided interventions. The puncture pathways were selected based on the $\mathrm{CT}$ results prior to the operation. The puncture site was verified using CT gantry laser lights and a landmark on the skin.

The co-axial technique was not used. The lung parenchyma was punctured using an $18 \mathrm{G}$ semiautomatic core needle (Precisa, Roma, Italy), and further CT scans were taken to adjust the location of the needle tip as appropriate. Once contact between the needle tip and $L N$ was made, 2 or more samples were retrieved from the nodule. The samples were stored in 10\% formaldehyde until further pathological investigation. The accumulated radiation dose was directly demonstrated on the CT device.

\section{Subjective imaging quality evaluation}

The image qualities were independently examined by 2 experienced thoracic radiologists, based on 4 levels. The levels were described as follows: A: good visualisation of the nodule and needle tip; $B$ : adequate visualisation of the nodule and needle tip; C: vague visualisation of the nodule and needle tip; and D: no visualisation of the nodule and needle tip [4]. A, C, or D evaluation was regarded as unsuitable for CT-guided CNB. Under these circumstances, the scanning parameters were adjusted to increase image quality and re-assess image quality evaluation. A re-evaluation of $\mathrm{C}$ or $\mathrm{D}$ constituted technical failure.

\section{Biopsy-based diagnoses}

Diagnoses based on biopsy were attributed to 1 of 4 categories: (a) malignant; (b) suspected malignant; (c) specific benign; or (d) non-specific benign. Specific benign lesions included both infection due to known microorganisms and benign tumours [10]. Non-specific benign lesions had benign inflammatory or fibrotic pathology, which could not lead to a definitive diagnosis [10].

\section{Final diagnoses}

Final malignant diagnosis could be made in 2 ways: (a) surgical resection; and (b) the biopsy-based diagnosis demonstrated a malignant lesion that could be considered as the conclusive diagnosis.

Final benign diagnosis could be made in 3 ways: (a) surgical resection; (b) the biopsy-based diagnosis demonstrated a specific benign, which could 
be considered as the conclusive diagnosis [10]; and (c) if the lesion decreased by $\geq 20 \%$ in size or remained stable (unchanged or decreased $<20 \%$ in size) for 12 months without any anticancer treatment on follow-up CT.

\section{Endpoints}

The primary endpoint was diagnostic performance. The secondary endpoints included technical success, radiation dose, and CNB-related complications.

CNB was considered as technically successful if sufficient samples were obtained from the lung lesion. Diagnostic performance included diagnostic yield and accuracy. Diagnostic yield was measured as CNB-based malignancies and specific benignities/all lesions, whereas diagnostic accuracy was calculated using precise diagnosis of the malignancy or benignity/all determinate results [2].

\section{Statistical analysis}

All statistical analyses were carried out using SPSS 16.0 software (SPSS Inc., Chicago, IL). Analysis of continuous variables was done through $t$-tests, and that of categorical data was done using $\chi^{2}$ tests. Inter-observer agreement of image quality was assessed by performing $\kappa$ analysis. A $\kappa$ value of $0.8-1$ was considered indicative of very good agreement; 0.6-0.79, good agreement; 0.4-0.59, moderate agreement; 0.2-0.39, fair agreement; and 0-0.19,

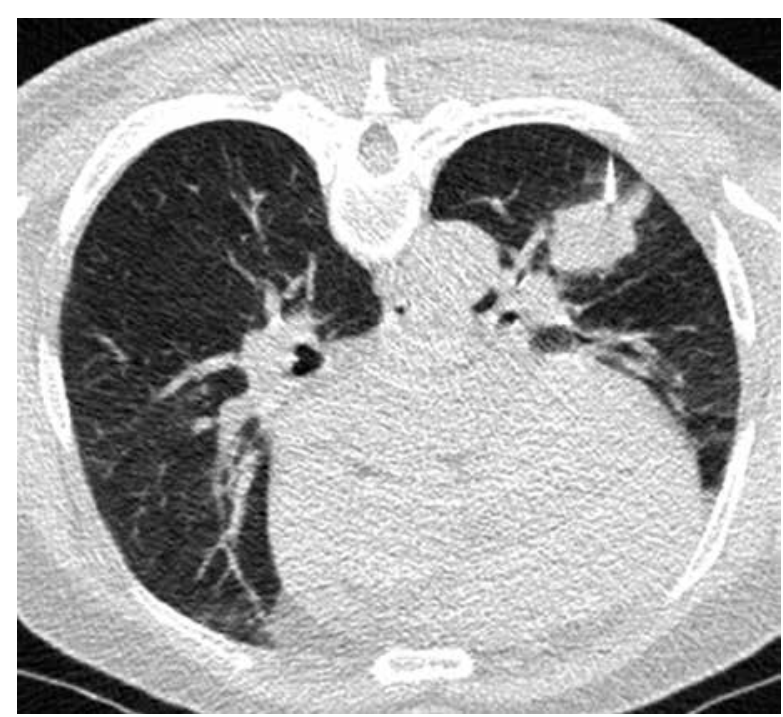

Photo 1. Low-dose computed tomography-guided core needle biopsy for lung nodule poor agreement. Predictors of diagnostic accuracy were assessed by logistic regression analysis. All variables that achieved a $p$-value of $<0.1$ in the initial univariate analysis were incorporated into the subsequent multivariate model. $P<0.05$ was considered statistically significant.

\section{Results}

\section{Primary data}

A total of 70 and 65 patients underwent LDCT-guided (Photo 1) and SDCT-guided (Photo 2) CNB procedures, respectively. The details of the baseline data are shown in Table I.

\section{Computed tomography-guided procedures}

Each patient underwent biopsy for 1 nodule. The technical success rates were $100 \%$ in both groups. The details of the CT-guided procedures are shown in Table II.

\section{Quality of images}

In the LDCT group, 55 (78.6\%) and 15 (21.4\%) images were classified as quality level $A$ and $B$, respectively. In SDCT group, all images were classified as quality level A ( $p<0.001 ; 100 \%$ vs. $78.6 \%$ ). None of the groups had images of level C or D. In both groups, inter-observer agreements were very good ( $\kappa$ value $=0.959$ and 1.000 , respectively).

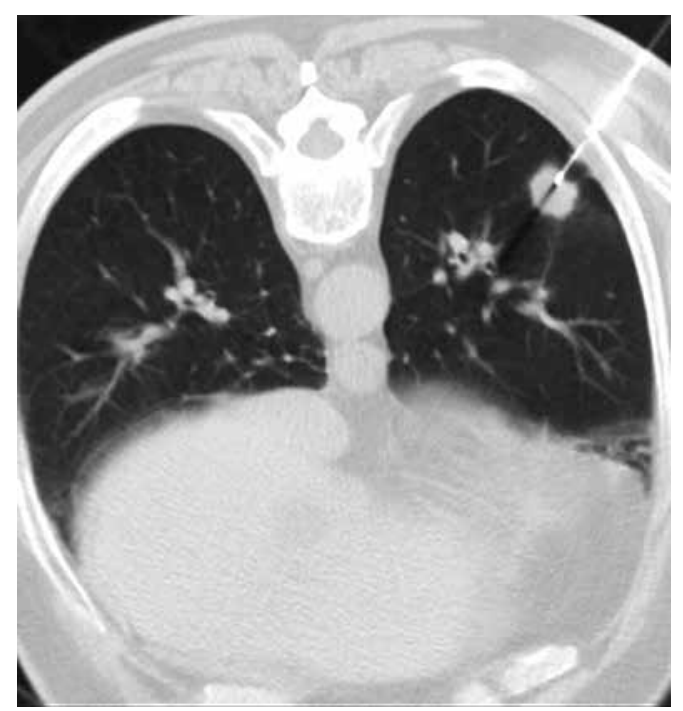

Photo 2. Standard-dose computed tomographyguided core needle biopsy for lung nodule 
Table I. Baseline data of patients and lung nodules

\begin{tabular}{|c|c|c|c|}
\hline Parameter & Low-dose group $(n=70)$ & Standard-dose group $(n=65)$ & $P$-value \\
\hline \multicolumn{4}{|l|}{ Patients' data: } \\
\hline Age [years] & $63.5 \pm 9.6$ & $59.9 \pm 12.8$ & 0.070 \\
\hline Gender (male/female), $n$ & $45 / 25$ & $39 / 26$ & 0.608 \\
\hline Smoking history, $n$ & 29 & 27 & 0.990 \\
\hline Tumour history, $n$ & 9 & 7 & 0.708 \\
\hline $\mathrm{BMI}\left[\mathrm{kg} / \mathrm{m}^{2}\right]$ & $23.1 \pm 3.3$ & $22.9 \pm 3.2$ & 0.794 \\
\hline \multicolumn{4}{|l|}{ LN features: } \\
\hline Diameter [mm] & $24.7 \pm 6.1$ & $23.6 \pm 6.8$ & 0.338 \\
\hline Side (left/right) & $34 / 36$ & $32 / 33$ & 0.939 \\
\hline Lobe (upper/non-upper) & $30 / 40$ & $27 / 38$ & 0.877 \\
\hline Lesion-pleura distance [mm] & $16.5 \pm 14.8$ & $20.4 \pm 17.6$ & 0.168 \\
\hline
\end{tabular}

$B M I$ - body mass index, $L N$ - lung nodule.

\section{Diagnostic accuracy}

\section{LDCT group}

The biopsy-based diagnoses included 45 malignant, 5 specific benign, and 20 non-specific benign lesions. All of the 5 specific benign lesions were tuberculosis.

The final diagnoses included 46 malignant and 22 benign lesions. Among the 20 biopsy-based non-specific benign lesions, 19 lesions were confirmed after CT follow-up $(n=11)$ or surgery $(n=8)$ as benign lesions, and 1 lesion was confirmed as malignancy by a second biopsy.
The diagnostic yield, sensitivity, specificity, and overall diagnostic accuracy were $71.4 \%, 97.8 \%, 100 \%$, and $98.6 \%$, respectively.

\section{SDCT group}

The biopsy-based diagnoses included 40 malignant, 1 suspected malignant, 4 specific benign, and 20 nonspecific benign lesions. The 4 specific benign lesions included hamartoma $(n=2)$ and tuberculosis $(n=2)$.

The final diagnoses included 44 malignant and 22 benign lesions. The 1 biopsy-based suspected malignant lesion was confirmed as adenocarcinoma after

Table II. Procedure details of the computed tomography-guided core needle biopsy

\begin{tabular}{|c|c|c|c|}
\hline Parameter & Low-dose group $(n=70)$ & Standard-dose group $(n=65)$ & $P$-value \\
\hline \multicolumn{4}{|l|}{ Biopsy procedure: } \\
\hline Technical success & $100 \%$ & $100 \%$ & - \\
\hline Needle-pleura angle (degrees) & $65.9 \pm 18.5$ & $66.5 \pm 17.4$ & 0.849 \\
\hline Prone/Supine/Decubitus & $36 / 25 / 9$ & $30 / 29 / 6$ & 0.533 \\
\hline Number of needle paths & $2.4 \pm 0.7$ & $2.4 \pm 0.6$ & 0.891 \\
\hline Number of samples & $2.1 \pm 0.4$ & $2.0 \pm 0.4$ & 0.199 \\
\hline Duration of procedure [min] & $11.7 \pm 4.5$ & $12.5 \pm 5.6$ & 0.379 \\
\hline Image quality (A/B/C/D) & $55 / 15 / 0 / 0$ & $65 / 0 / 0 / 0$ & $<0.001$ \\
\hline \multicolumn{4}{|l|}{ Complications, $n$ : } \\
\hline Pneumothorax & 9 & 12 & 0.369 \\
\hline Lung haemorrhage & 11 & 12 & 0.671 \\
\hline \multicolumn{4}{|l|}{ Radiation exposure $[\mathrm{mGy} \cdot \mathrm{cm}]$ : } \\
\hline DLP & $38.3 \pm 17.0$ & $376.0 \pm 118.7$ & $<0.001$ \\
\hline
\end{tabular}


Table III. Diagnostic performance of computed tomography-guided core needle biopsy

\begin{tabular}{|c|c|c|c|}
\hline Parameter & Low-dose group $(n=70)$ & Standard-dose group $(n=65)$ & $P$-value \\
\hline Biopsy pathological diagnosis, $n$ : & & & 0.748 \\
\hline Malignancy & 45 & 40 & \\
\hline Suspected malignancy & 0 & 1 & \\
\hline Specific benign & 5 & 4 & \\
\hline Non-specific benign & 20 & 20 & \\
\hline Final diagnosis, $n$ : & & & 0.808 \\
\hline Malignancy & 46 & 44 & \\
\hline Benign & 24 & 21 & \\
\hline \multicolumn{4}{|l|}{ Diagnostic performance, $n(\%)$ : } \\
\hline Diagnostic yield & $50 / 70(71.4 \%)$ & $44 / 65(67.7 \%)$ & 0.637 \\
\hline Sensitivity & $45 / 46(97.8 \%)$ & $41 / 44(93.2 \%)$ & 0.625 \\
\hline Specificity & 24/24 (100\%) & $21 / 21(100 \%)$ & - \\
\hline Overall accuracy & $69 / 70(98.6 \%)$ & $62 / 65(95.4 \%)$ & 0.560 \\
\hline
\end{tabular}

surgery. Among the 20 biopsy-based non-specific benign lesions, 17 were confirmed as benign according to CT follow-up ( $n=13)$ or surgical results $(n=4)$, and 3 lesions were confirmed as malignancy by surgery.

The diagnostic yield, specificity, sensitivity, and overall diagnostic accuracy were $67.7 \%, 100 \%$, $93.2 \%$, and $95.4 \%$, respectively.

There was no significant difference in diagnostic performance between the LDCT and SDCT groups (Table III).

\section{Predictors of diagnostic accuracy}

In univariate logistic analysis, the risk factors of diagnostic failure included older age ( $p=0.05 ; 95 \%$ $\mathrm{Cl}: 1.000-1.317)$, larger needle-pleura angle ( $p=$ 0.094; 95\% Cl: 0.980-1.294), and small number of tissue samples ( $p=0.01 ; 95 \% \mathrm{Cl}: 0.032-0.625)$. In multivariate analysis, the independent risk factor of diagnostic failure was a small number of tissue samples ( $p=0.012 ; 95 \% \mathrm{Cl}$ : 0.033-0.651).

\section{Complications}

Pneumothorax developed in 9 and 12 patients in the LDCT and SDCT groups, respectively (12.9\% vs. $18.5 \%, p=0.369)$. Among them, only 2 patients in the LDCT group required chest drainage.

Lung haemorrhage developed in 11 and 12 patients in the LDCT and SDCT groups, respectively (15.7\% vs. $18.5 \%, p=0.671)$. All of these patients were treated by haemostasis.

\section{Radiation dose}

The mean dose-length product (DLP) was 38.3 $\pm 17.0 \mathrm{mGy} \cdot \mathrm{cm}$ and $376.0 \pm 118.7 \mathrm{mGy} \cdot \mathrm{cm}$ in the LDCT and SDCT groups, respectively $(p<0.001)$.

\section{Subgroup analysis}

A subgroup analysis of small $(\leq 20 \mathrm{~mm})$ LNs was performed (Table IV). There was no significant difference in diagnostic yield (72.2\% vs. $72 \%, p=0.987)$, sensitivity (100\% vs. $94.4 \%, p=1.000)$, and overall accuracy (100\% vs. $96 \%, p=1.000)$ between the 2 groups. The complication rates were comparable between the 2 groups. The mean DLP was 39.9 $\pm 14.4 \mathrm{mGy} \cdot \mathrm{cm}$ and $399.1 \pm 120.8 \mathrm{mGy} \cdot \mathrm{cm}$ in the LDCT and SDCT groups, respectively $(p<0.001)$.

\section{Discussion}

In this study, we compared the feasibility, diagnostic performance, and radiation exposure between LDCT-guided and SDCT-guided CNB for LNs. Compared to the previous studies regarding LDCT-guided CNB for all lung lesions [4, 5, 7, 8], this study only focused on LNs.

The technical successful rate was $100 \%$ in both groups, although quality level A images were found significantly more in the SDCT group. In addition, the procedure time was similar between the 2 groups. Some previous studies also found that SDCT showed higher image quality than did LDCT, but without 
Table IV. Subgroup analysis for small ( $\leq 20 \mathrm{~mm}$ ) lung nodules

\begin{tabular}{|lccc|}
\hline Parameter & Low-dose group $(n=18)$ & Standard-dose group $(n=25)$ & $P$-value \\
\hline Diagnostic performance, $n(\%):$ & & & \\
\hline Diagnostic yield & $13 / 18(72.2 \%)$ & $18 / 25(72.0 \%)$ & 0.987 \\
\hline Sensitivity & $11 / 11(100 \%)$ & $17 / 18(94.4 \%)$ & 1.000 \\
\hline Specificity & $7 / 7(100 \%)$ & $7 / 7(100 \%)$ & 1.000 \\
\hline Overall accuracy & $18 / 18(100 \%)$ & $24 / 25(96 \%)$ & 1.000 \\
\hline \begin{tabular}{l} 
Complications, $n:$ \\
\hline Pneumothorax
\end{tabular} & 2 & 2 & 0.452 \\
\hline Lung haemorrhage & 7 & 7 & $<0.001$ \\
\hline Radiation dose $[\mathrm{mGy} \cdot \mathrm{cm}]:$ & $39.9 \pm 14.4$ & $399.1 \pm 120.8$ & \\
\hline DLP & & & \\
\hline
\end{tabular}

$D L P$ - dose-length product.

a significant difference in technical successful rate $[4,7]$. These data suggest that LDCT could provide adequate clarity for a CT-guided CNB procedure. Unlike the conventional imaging diagnosis, CT-guided CNB does not require intricate imaging details, but only the adequate visualisation of the lesion and needle tip, to be successful.

Diagnostic performance was the chief endpoint of CT-guided CNB for LNs in our study. We demonstrated comparable diagnostic yield, sensitivity, and overall accuracy of diagnosis between the LDCT and SDCT groups. These comparative results are in consistent with previous studies regarding LDCT-guided biopsy [4-8]. Moreover, the overall accuracy in diagnosis and sensitivity in both groups are also comparable to those in many previous studies regarding CT-guided LN biopsy $[1,2,6]$. These results indicated that LDCT did not alter the diagnostic accuracy of CNB.

At present, surgical resection is widely used for the management of LNs $[11,12]$. The types of surgical resection contained sublobar resection and lobectomy [11, 12]. Lobectomy should be performed for invasive lung cancers, and sublobar resection can be performed for cancer in situ [11, 12]. Therefore, the preoperative CT-guided CNB results play an important guiding role in the choice of surgery.

Of the LNs, 36\% were diagnosed as benign based on the CNB in this study. The CNB-based benign results were also important to surgeons. If the CNB indicated specific benign results, these could be accepted as the final diagnosis and surgical resection could be avoided [7]. For the non-specific benign results, regular CT follow-up could be performed first; if the LN decreased or remained stable for 12 months, the surgical resection could also be avoided [7].

According to our logistic analysis results, the independent risk factor of diagnostic failure in a CT-guided CNB procedure was small a number of tissue samples. This result is consistent with the previous studies regarding CT-guided CNB for LNs [1, 2]. Therefore, obtaining more samples may be necessary during CT-guided CNB procedures. In Lee's study [5], more than 75\% of cases were obtained with 2-3 tissue samples. Some researchers also found that procedure-related pneumothorax may cause diagnostic failure [7]. It was not demonstrated in the present study, which may be attributed to our limited patient sample size.

In addition, our diagnostic performances were similar to previous studies examining CT fluoroscopyguided, cone-beam CT (CBCT)-guided, or other novel technique-assisted CT-guided lung biopsy [3, 10, $13,14]$. These results indicate that LDCT-guided CNB was a highly accurate diagnostic method for LNs. In addition, these findings also indicate that CT fluoroscopy-guided and CBCT-guided lung biopsy may not effectively increase the diagnostic accuracy when compared to normal CT-guided lung biopsy.

The procedure-related complications, both pneumothorax and lung haemorrhage, were similar between the 2 groups. These findings indicate that reducing radiation dose not increase the risk of CNB-associated complications [7]. Many previous studies have described CT fluoroscopy-guided or CBCT-guided lung biopsy to have fewer procedural complications [3, 10, 13]. However, both of these procedures expose both radiologists and patients to significantly more radiation than conventional CT-guid- 
ed lung biopsy [10]. Furthermore, most biopsy-related complications were self-limited [15]. Thus, LDCT-guided CNB was a safe diagnostic method for LNs.

Furthermore, by using LDCT, the radiation exposure can be brought down by $90 \%$ as compared to SDCT, by reducing $90 \%$ of the tube current. In another study, Lee et al. [5] were able to achieve a 50\% reduction in radiation exposure by decreasing the tube voltage during CT-guided CNB. Yet another researcher conducted magnetic resonance (MR)-guided CNB for LNs without any radiation [16]. However, MR-guided lung biopsy is very costly and requires a longer duration of scanning, which may introduce a higher incidence of complications.

We also performed a subgroup analysis focusing on small LNs. Based on our results, the diagnostic performance and complication rates between the LDCT and SDCT groups were comparable. However, the DLP was significantly reduced in the LDCT group as compared to the SDCT group. Furthermore, we demonstrated a 100\% diagnostic accuracy of LDCT-guided CNB for small LNs. However, we recognise that our patient sample size was small (18 LDCT and 25 SDCT patients). Therefore, further clinical trials with a large sample population are warranted.

This study has certain limitations. Firstly, the study was retrospective in nature, and to further establish the results prospective randomised trials are needed in the future. Secondly, the sample size was small. Thirdly, no unified criteria were followed for the number of samples required for recruitment in the study. Instead, the samples were obtained in accordance with our experience, which may have caused further bias.

\section{Conclusions}

Compared to SDCT-guided CNB, LDCT-guided CNB can provide comparable safety and similar diagnostic performance for patients with LNs while dramatically reducing the radiation exposure.

\section{Conflict of interest}

The authors declare no conflict of interest.

\section{References}

1. Li Y, Du Y, Yang HF, et al. CT-guided percutaneous core needle biopsy for small ( $\leq 20 \mathrm{~mm})$ pulmonary lesions. Clin Radiol 2013; 68: e43-e48.
2. Li Y, Wang T, Fu YF, et al. Computed tomography-guided biopsy for sub-centimetre lung nodules: technical success and diagnostic accuracy. Clin Respir J 2020; 14: 605-10.

3. Choo JY, Park CM, Lee NK, et al. Percutaneous transthoracic needle biopsy of small $(\leq 1 \mathrm{~cm})$ lung nodules under $C$-arm conebeam CT virtual navigation guidance. Eur Radiol 2013; 23: 712-9.

4. Meng XX, Kuai XP, Dong WH, et al. Comparison of lung lesion biopsies between low-dose CT-guided and conventional CTguided techniques. Acta Radiol 2013; 54: 909-15.

5. Lee HN, Lee SM, Choe J, et al. Diagnostic performance of CT-guided percutaneous transthoracic core needle biopsy using low tube voltage (100 kVp): comparison with conventional tube voltage (120 kVp). Acta Radiol 2018; 59: 425-33.

6. Li C, Liu B, Meng $\mathrm{H}$, et al. Efficacy and radiation exposure of ultra-low-dose chest CT at $100 \mathrm{kVp}$ with tin filtration in CT-guided percutaneous core needle biopsy for small pulmonary lesions using a third-generation dual-source CT scanner. J Vasc Interv Radiol 2019; 30: 95-102.

7. Fu YF, Li GC, Xu QS, et al. Computed tomography-guided lung biopsy: a randomized controlled trial of low-dose versus standard-dose protocol. Eur Radiol 2020; 30: 1584-92.

8. Frisch BK, Slebocki K, Mammadov K, et al. Implementation of ultra-low dose lung protocols in CT-guided lung biopsies: feasibility and safety in the clinical setting. J Int Med Res 2017; 45: 2101-9.

9. Ettinger DS, Wood DE, Akerley W, et al. Non-small cell lung cancer, Version 6.2015. J Natl Compr Canc Netw 2015; 13: 515-24.

10. Kim GR, Hur J, Lee SM, et al. CT fluoroscopy-guided lung biopsy versus conventional CT-guided lung biopsy: a prospective controlled study to assess radiation doses and diagnostic performance. Eur Radiol 2011; 21: 232-9.

11. Zhong L, Hu W, Li S, et al. Clinical study of video-assisted thoracoscopic surgery wedge resection in early-stage lung cancer by tumor mapping with indocyanine green. Videosurgery Miniinv 2019; 14: 545-50.

12. Zhang ZD, Wang HL, Liu XY, et al. Methylene blue versus coilbased computed tomography-guided localization of lung nodules. Thorac Cardiovasc Surg 2020; 68: 540-4.

13. Rotolo N, Floridi C, Imperatori A, et al. Comparison of conebeam CT-guided and CT fluoroscopy-guided transthoracic needle biopsy of lung nodules. Eur Radiol 2016; 26: 381-9.

14. Yaffe D, Koslow M, Haskiya $H$, et al. A novel technique for CT-guided transthoracic biopsy of lung lesions: improved biopsy accuracy and safety. Eur Radiol 2015; 25: 3354-60.

15. Heerink WJ, de Bock GH, de Jonge GJ, et al. Complication rates of CT-guided transthoracic lung biopsy: meta-analysis. Eur Radiol 2017; 27: 138-48.

16. Liu S, Li C, Yu X, et al. Diagnostic accuracy of MRI-guided percutaneous transthoracic needle biopsy of solitary pulmonary nodules. Cardiovasc Intervent Radiol 2015; 38: 416-21.

Received: 1.01.2021, accepted: 11.01.2021. 